\title{
Sliding-mode Observer Design for Wheel Speed of Sensorless Distributed Driven Electric Vehicles
}

\author{
X. Z. Zhang ${ }^{1,2}$ \\ 1) The CIC of Hunan Institute of Engineering, Xiangtan 411104, China \\ ${ }^{2)}$ School of Computer and Communication, Hunan Institute of Engineering, \\ Xiangtan 411104, China \\ z_x_z2000@163.com
}

\begin{abstract}
This paper presented a novel sliding-mode observer for wheel speed, instead of the conventional speed sensors, of the distributed-driven electric vehicle with in-wheel motors. By selecting the stator current and the rotor flux as the state variables, the current error dynamics are derived from the in-wheel motor mathematical model in stationary coordinate system. With the designed control gain big enough to make the error system enter the sliding mode, the wheel speed observation is deduced after the observed current and flux converges. The observation is obtained directly from the current estimation error and the flux estimation without any integral process. Compared with conventional SMO, the proposed scheme is more simple, practical and reliable. An experimental test was carried out to verify the effectiveness of the proposed design. Results show that the observer can effectively estimate the wheel speed with high accuracy as well as its fast convergence rate online-ly.
\end{abstract}

Keywords: distributed-driven electric vehicle, wheel speed sensor, sliding-mode observer, sensorless control

\section{Introduction}

Recently, putting the motor in wheel inside and each wheel with a motor which is placed as driving source of new distributed drive electric vehicle has attracted wide attention. In distributed drive, the degree of freedom of motion for each wheel increase, each motor drive/brake torque can be controlled independently. Car running state control is directly applied to control the motor just combined with vehicle running status, it is possible to accurately control the distribution of the drive/brake torque of each wheel, which can be more flexible and efficient implementation of a variety of vehicle control. In such kinds of electronic control system of automobile brake/drive as Anti-lock Braking System(ABS), Acceleration Slip Regulation(ASR), Cornering Braking Control(CBC), Electronic Stability Program(ESP), which all need to get the wheel speed information.

Currently, the wheel speed information mostly comes from the wheel speed sensor monitoring of wheel speed directly, and all of the wheel speed must be tested by sensor. Magnetic-electric Wheel-speed Sensor used in conventional car has the weakness of bulky, heavy quality, output voltage amplitude of the sensor varying with size of the speed and poor resistance to electromagnetic interference fault. So the sensors are not suitable for direct move to a distributed drive electric vehicles. Typical ABS wheel speed sensor's diameter range is of $44-115 \mathrm{~mm}$, the width of the range of $6-30 \mathrm{~mm}$, difficult to integrate into the space of the already narrow in electric vehicle's in-wheel motors. Due to the influence of noise, ABS sensor's accuracy at idle $(<5 \mathrm{~km} / \mathrm{h})$ under the anti-lock brake control is difficult to meet the needs; direct contacting with the wheel and the road surface, dust, rainwater and other debris which can contaminate the sensor and even lead failure. 
On the other hand, with the development of modern control theory, speed sensorless technology has low cost, high reliability, maintenance-free and so on merits and widely studied. In it, based on the state estimation observer, this can be used for real-time observation in various states and parameters of vehicle system, because of its simple structure and widely used. The sliding mode observer for the perturbation of parameter, the modeling error and external disturbance and so on has good robustness, especially in the vehicle control system has been widely applied.

For distributed drive electric vehicle of installing in-wheel motors, this paper presents a new wheel speed observer which is designed to replace the traditional wheel speed sensor. So using sliding mode speed observation technology design of wheel speed observer, compared to traditional sliding mode observer must exist in the speed of integral part, the observer of this paper is more simple, practical and reliable. An experimental test was carried out to verify the effectiveness of the proposed design. The results show that the observer can effectively estimate the wheel speed with high accuracy as well as its fast convergence rate onlinely.

\section{Design of Sliding-Mode Observer for Wheel Speed}

\subsection{The Mathematical Model of the In-Wheel Motor}

Distributed drive electric vehicles put a in-wheel induction motor inside each wheel. By selecting the stator current and the rotor flux as the state variables, the equation of state of the motor is represented by Equation (1) in the two-phase stationary coordinate system.

$$
\left\{\begin{array}{l}
\dot{\psi}_{\mathrm{r} \alpha}=-\frac{1}{\tau_{\mathrm{r}}} \psi_{\mathrm{r} \alpha}+\frac{L_{\mathrm{m}}}{\tau_{\mathrm{r}}} i_{\mathrm{s} \alpha}-n_{\mathrm{p}} \omega_{\mathrm{rm}} \psi_{\mathrm{r} \beta} \\
\dot{\psi}_{\mathrm{r} \beta}=-\frac{1}{\tau_{\mathrm{r}}} \psi_{\mathrm{r} \beta}+\frac{L_{\mathrm{m}}}{\tau_{\mathrm{r}}} i_{\mathrm{s} \beta}+n_{\mathrm{p}} \omega_{\mathrm{rm}} \psi_{\mathrm{r} \alpha} \\
\dot{i}_{\mathrm{s} \alpha}=-\gamma i_{\mathrm{s} \alpha}+\frac{k_{\mathrm{s}}}{\tau_{\mathrm{r}}} \psi_{\mathrm{r} \alpha}+k_{\mathrm{s}} n_{\mathrm{p}} \omega_{\mathrm{rm}} \psi_{\mathrm{r} \beta}+\frac{1}{\sigma L_{\mathrm{s}}} u_{\mathrm{s} \alpha} \\
\dot{i}_{\mathrm{s} \beta}=-\gamma i_{\mathrm{s} \beta}-k_{\mathrm{s}} n_{\mathrm{p}} \omega_{\mathrm{rm}} \psi_{\mathrm{r} \alpha}+\frac{k_{\mathrm{s}}}{\tau_{\mathrm{r}}} \psi_{\mathrm{r} \beta}+\frac{1}{\sigma L_{\mathrm{s}}} u_{\mathrm{s} \beta} \\
\dot{\omega}_{\mathrm{rm}}=\mu\left(\psi_{\mathrm{s} \alpha} i_{\mathrm{s} \beta}-\psi_{\mathrm{s} \beta} i_{\mathrm{s} \alpha}\right)-\frac{T_{\mathrm{L}}}{J}-\frac{B}{J} \omega_{\mathrm{rm}}
\end{array}\right.
$$

where $\mathrm{x}=\left[\psi_{\mathrm{r} \alpha}, \psi_{\mathrm{r} \beta}, \mathrm{i}_{\mathrm{s \alpha}}, \mathrm{i}_{\mathrm{s \beta}}, \omega_{\mathrm{rm}}\right]^{\mathrm{T}}, \mathrm{L}_{\mathrm{m}}$ is mutual inductance of in-wheel motor, $\mathrm{L}_{\mathrm{r}}$ is the rotor inductance, $\mathrm{L}_{\mathrm{s}}$ is the stator inductance, $\mathrm{R}_{\mathrm{r}}$ is the rotor resistance, $\mathrm{R}_{\mathrm{s}}$ is the stator resistance, $\mathrm{J}$ is moment of inertia, $\mathrm{np}$ is number of motor poles, $\psi_{\mathrm{s} \alpha}$ and $\psi_{\mathrm{s} \beta}$ respectively represent $x$-coordinate and $y$-coordinate of the stator flux, $i_{s \alpha}$ and $i_{s \beta}$ respectively represent $\mathrm{x}$-coordinate and $\mathrm{y}$-coordinate of the stator current, $\mathrm{u}_{\mathrm{s} \alpha}$ and $\mathrm{u}_{\mathrm{s} \beta}$ respectively represent $\mathrm{x}$-coordinate and $\mathrm{y}$-coordinate of the stator voltage, $\omega_{\mathrm{rm}}$ and $\omega_{\mathrm{r}}$ respectively represent the rotor mechanical angular velocity and electric angular velocity, Tm is load torque. Also, there exist : $\sigma=1-\frac{\mathrm{L}_{\mathrm{m}}^{2}}{\mathrm{~L}_{\mathrm{s}} \mathrm{L}_{\mathrm{r}}}, \tau_{\mathrm{r}}=\frac{\mathrm{L}_{\mathrm{r}}}{\mathrm{R}_{\mathrm{r}}}, \mathrm{k}_{\mathrm{s}}=\frac{\mathrm{L}_{\mathrm{m}}}{\sigma \mathrm{L}_{\mathrm{s}} \mathrm{L}_{\mathrm{r}}}, \gamma=\frac{\mathrm{L}_{\mathrm{s}} \mathrm{R}_{\mathrm{r}}+\mathrm{L}_{\mathrm{r}} \mathrm{R}_{\mathrm{s}}}{\sigma \mathrm{L}_{\mathrm{s}} \mathrm{L}_{\mathrm{r}}}, \mu=\frac{\mathrm{n}_{\mathrm{p}} \mathrm{L}_{\mathrm{m}}}{J \mathrm{~L}_{\mathrm{r}}}$.

Equation (1) can be rewritten as the following matrix form : 


$$
\begin{aligned}
& {\left[\begin{array}{l}
i_{\mathrm{s} \alpha} \\
\dot{i}_{\mathrm{s} \beta}
\end{array}\right]=-\gamma\left[\begin{array}{l}
i_{\mathrm{s} \alpha} \\
i_{\mathrm{s} \beta}
\end{array}\right]+k_{\mathrm{s}}\left[\begin{array}{cc}
1 / \tau_{\mathrm{r}} & \omega_{\mathrm{r}} \\
-\omega_{\mathrm{r}} & 1 / \tau_{\mathrm{r}}
\end{array}\right]\left[\begin{array}{l}
\psi_{\mathrm{ra}} \\
\psi_{\mathrm{r \beta}}
\end{array}\right]+\frac{1}{\sigma L_{\mathrm{s}}}\left[\begin{array}{l}
u_{\mathrm{s} \alpha} \\
u_{\mathrm{s} \beta}
\end{array}\right]} \\
& {\left[\begin{array}{l}
\dot{\psi}_{\mathrm{r} \alpha} \\
\dot{\psi}_{\mathrm{r} \beta}
\end{array}\right]=\left[\begin{array}{cc}
-1 / \tau_{\mathrm{r}} & -\omega_{\mathrm{r}} \\
\omega_{\mathrm{r}} & -1 / \tau_{\mathrm{r}}
\end{array}\right]\left[\begin{array}{l}
\psi_{\mathrm{r} \alpha} \\
\psi_{\mathrm{r} \beta}
\end{array}\right]+\frac{L_{\mathrm{m}}}{\tau_{\mathrm{r}}}\left[\begin{array}{c}
i_{\mathrm{s} \alpha} \\
i_{\mathrm{s} \beta}
\end{array}\right]}
\end{aligned}
$$

It follows from (2), in-wheel motor stator current and rotor flux variables coupled to each other, in order to achieve the accurate observation of wheel speed, you first need to separate these two ones.

\subsection{Sliding-Mode Observer for Wheel Speed}

After obtaining the in-wheel motor stator voltage and current components measured, according to the above model, the design is based on sliding mode observer to estimate the rotor actual wheel speed. Since the stator current is measurable, and the rotor flux is difficult to measure, so it separates $\psi_{\mathrm{r} \alpha}$ and $\psi_{\mathrm{r} \beta}$ from $i_{s \alpha}$ and $i_{s \beta}$ in the state equation, the wheel speed sliding mode observer is constructed as follows:

$$
\begin{aligned}
& {\left[\begin{array}{l}
\dot{\hat{i}}_{\mathrm{s} \alpha} \\
\hat{\hat{i}}_{\mathrm{s} \beta}
\end{array}\right]=-\gamma\left[\begin{array}{l}
\hat{i}_{\mathrm{s} \alpha} \\
\hat{i}_{\mathrm{s} \beta}
\end{array}\right]+k_{\mathrm{s}}\left[\begin{array}{l}
u_{\mathrm{r} \alpha}^{\mathrm{s}} \\
u_{\mathrm{r} \beta}^{\mathrm{s}}
\end{array}\right]+\frac{1}{\sigma L_{\mathrm{s}}}\left[\begin{array}{l}
u_{\mathrm{s} \alpha} \\
u_{\mathrm{s} \beta}
\end{array}\right]} \\
& {\left[\begin{array}{l}
\dot{\hat{\psi}}_{\mathrm{r} \alpha} \\
\dot{\hat{\psi}}_{\mathrm{r} \beta}
\end{array}\right]=\left[\begin{array}{cc}
-1 / \tau_{\mathrm{r}} & -\hat{\omega}_{\mathrm{r}} \\
\hat{\omega}_{\mathrm{r}} & -1 / \tau_{\mathrm{r}}
\end{array}\right]\left[\begin{array}{l}
\hat{\psi}_{\mathrm{r} \alpha} \\
\hat{\psi}_{\mathrm{r} \beta}
\end{array}\right]+\frac{L_{\mathrm{m}}}{\hat{\tau}_{\mathrm{r}}}\left[\begin{array}{l}
i_{\mathrm{s} \alpha} \\
i_{\mathrm{s} \beta}
\end{array}\right]}
\end{aligned}
$$

where the superscript " $\wedge$ " indicates the observed value of the corresponding variable, namely: $\hat{\psi}_{\mathrm{r} \alpha}$ and $\hat{\psi}_{\mathrm{r} \beta}$ respectively represent $\mathrm{x}$-coordinate and y-coordinate of the rotor flux observed value, $\hat{\mathrm{i}}_{\mathrm{s} \alpha}$ and $\hat{\mathrm{i}}_{\mathrm{s} \beta}$ are stator current observed values, $\hat{\omega}_{\mathrm{r}}$ is wheel speed estimated value.

$\mathrm{u}_{\mathrm{r} \alpha}^{\mathrm{s}}$ and $\mathrm{u}_{\mathrm{r} \beta}^{\mathrm{s}}$ are the sliding mode, defined as follows:

$$
\begin{aligned}
& u_{\mathrm{r} \alpha}^{\mathrm{s}}=-h_{1} \operatorname{sgn}\left(\tilde{i}_{\mathrm{s} \alpha}\right) \\
& u_{\mathrm{r} \beta}^{\mathrm{s}}=-h_{2} \operatorname{sgn}\left(\tilde{i}_{\mathrm{s} \beta}\right)
\end{aligned}
$$

where the superscript " $\sim$ " indicates the deviation between the observed value and the actual value, namely: stator current observational error are $\tilde{\mathrm{i}}_{\mathrm{s} \alpha}=\hat{\mathrm{i}}_{\mathrm{s} \alpha}-\mathrm{i}_{\mathrm{s} \alpha}$ and $\tilde{\mathrm{i}}_{\mathrm{s} \beta}=\hat{\mathrm{i}}_{\mathrm{s} \beta}-\mathrm{i}_{\mathrm{s} \beta}, \mathrm{h} 1$ and $\mathrm{h} 2$ are undetermined observations gain, $\operatorname{sgn}()$ is sign function.

In the formula (3), the current observation equation does not depend on the magnetic linkage, but depends only on the portion of the stator voltage and sliding mode observer. With the observation Equation (3) minus induction motor state Equation (2), we can get the stator current observer error dynamic equation is:

$$
\left[\begin{array}{l}
\dot{\tilde{i}}_{\mathrm{su}} \\
\dot{\tilde{i}}_{s \beta}
\end{array}\right]=-k_{\mathrm{s}}\left[\begin{array}{c}
\psi_{\mathrm{ru}} / \tau_{\mathrm{r}}+\omega_{\mathrm{r}} \psi_{\mathrm{r} \beta} \\
-\omega_{\mathrm{r}} \psi_{\mathrm{ru}}+\psi_{\mathrm{r} \beta} / \tau_{\mathrm{r}}
\end{array}\right]+\left[\begin{array}{c}
u_{\mathrm{ra}}^{\mathrm{s}} \\
u_{\mathrm{r} \beta}^{s}
\end{array}\right]-\gamma\left[\begin{array}{c}
\tilde{i}_{\mathrm{s} \alpha} \\
\tilde{i}_{\mathrm{s} \beta}
\end{array}\right]
$$

Sliding mode variable for stator current observation deviation, sliding mode function is designed as follows:

$$
S=\left[\tilde{i}_{\mathrm{s} \alpha}, \tilde{i}_{\mathrm{s} \beta}\right]^{T}
$$

Lyapunov function is assumed $V=S^{2}$ and $\dot{V}$ satisfies the following conditions:

$$
\dot{V}=\dot{S}^{T} S=\left[\begin{array}{ll}
\dot{\tilde{i}}_{\mathrm{s} \alpha} & \dot{\tilde{i}_{\mathrm{s} \beta}}
\end{array}\right] \cdot\left[\begin{array}{ll}
\tilde{i}_{\mathrm{s} \alpha} & \tilde{i}_{\mathrm{s} \beta}
\end{array}\right]^{T}<0
$$


That is, when $\tilde{\mathrm{i}}_{\mathrm{s} \alpha} \dot{\overrightarrow{\mathrm{i}}}_{\mathrm{s} \alpha}<0$ and $\tilde{\mathrm{i}}_{\mathrm{s} \beta} \dot{\tilde{\mathrm{i}}}_{\mathrm{s} \beta}<0$ are satisfied sliding mode reaching condition, sliding mode observer enters into the sliding mode, which can be deduced as such:

$$
\begin{aligned}
& \tilde{i}_{\mathrm{s} \alpha}\left[-h_{1} \operatorname{sgn}\left(\tilde{i}_{\mathrm{s} \alpha}\right)-\gamma \tilde{i}_{\mathrm{s} \alpha}-\frac{k_{\mathrm{s}}}{\tau_{\mathrm{r}}} \psi_{\mathrm{r} \alpha}-k_{\mathrm{s}} \omega_{\mathrm{r}} \psi_{\mathrm{r} \beta}\right]<0 \\
& \tilde{i}_{\mathrm{s} \beta}\left[-h_{1} \operatorname{sgn}\left(\tilde{i}_{\mathrm{s} \beta}\right)-\gamma \tilde{i}_{\mathrm{s} \beta}-\frac{k_{\mathrm{s}}}{\tau_{\mathrm{r}}} \psi_{\mathrm{r} \beta}+k_{\mathrm{s}} \omega_{\mathrm{r}} \psi_{\mathrm{r} \alpha}\right]<0
\end{aligned}
$$

By the inequality (8) can be derived to control the gain of $h_{1}$ and $h_{2}$ values must meet the following requirements:

$$
\begin{aligned}
& h_{1}>\left|-\gamma \tilde{i}_{\mathrm{s} \alpha}-k_{\mathrm{s}}\left(\psi_{\mathrm{r} \alpha} \tau_{\mathrm{r}}^{-1}+\omega_{\mathrm{r}} \psi_{\mathrm{r} \beta}\right)\right| \\
& h_{2}>\left|-\lambda \tilde{i}_{\mathrm{s} \beta}-k_{\mathrm{s}}\left(\psi_{\mathrm{r} \beta} \tau_{\mathrm{r}}^{-1}-\omega_{\mathrm{r}} \psi_{\mathrm{r} \alpha}\right)\right|
\end{aligned}
$$

Choosing big enough $\mathrm{h} 1$ and $\mathrm{h} 2$, when the formula (9) and the inequality (7) hold, the system enters into the sliding mode $S=0$, then the stator current observational error is zero, thus sliding mode observer is stable. Because $\mathrm{h} 1$ and $\mathrm{h} 2$ is big enough, a portion of the sliding mode observer is a large amplitude switching input, including a very rich highfrequency component of unnecessary damage to power electronic devices. The engineering practice is to reduce the high-frequency component of the damage to power electronic devices using saturation function instead of sign function.

After the observer is stable, observed currents $\hat{\mathrm{i}}_{\mathrm{s} \alpha}, \hat{\mathrm{i}}_{\mathrm{s} \beta}$ and actual currents $i_{\mathrm{s} \alpha}, \mathrm{i}_{\mathrm{s} \beta}$ are equal. By formula (3) can be obtained:

$$
\left[\begin{array}{c}
u_{\mathrm{r} \alpha}^{\mathrm{s}} \\
u_{\mathrm{r} \beta}^{\mathrm{s}}
\end{array}\right]=\left[\begin{array}{cc}
1 / \tau_{\mathrm{r}} & \hat{\omega}_{\mathrm{r}} \\
-\hat{\omega}_{\mathrm{r}} & 1 / \tau_{\mathrm{r}}
\end{array}\right]\left[\begin{array}{l}
\hat{\psi}_{\mathrm{r} \alpha} \\
\hat{\psi}_{\mathrm{r} \beta}
\end{array}\right]
$$

Analyze the formula (3) flux measurement equation and Equation (10), we can found formula (3), it's the first coefficient matrix of the right can be made of the sliding mode observation replacement, namely:

$$
\left[\begin{array}{c}
\dot{\hat{\psi}}_{\mathrm{r} \alpha} \\
\dot{\hat{\psi}}_{\mathrm{r} \beta}
\end{array}\right]=-\left[\begin{array}{c}
u_{\mathrm{r} \alpha}^{\mathrm{s}} \\
u_{\mathrm{r} \beta}^{\mathrm{s}}
\end{array}\right]+\frac{L_{\mathrm{m}}}{\tau_{\mathrm{r}}}\left[\begin{array}{c}
i_{\mathrm{s} \alpha} \\
i_{\mathrm{s} \beta}
\end{array}\right]
$$

In formula (11) does not contain any wheel speed information, once the stator current gets an accurate estimation, the flux observations $\hat{\psi}_{\mathrm{r} \alpha}$ and $\hat{\psi}_{\mathrm{r} \beta}$ will converge to the actual value $\psi_{\mathrm{r} \alpha}$ and $\psi_{\mathrm{r} \beta}$. Represented by the formula (10), which can be solved wheel speed estimated value $\hat{\omega}_{\mathrm{r}}$. Rewriting the formula (10) as follows:

$$
\left[\begin{array}{c}
u_{\mathrm{r} \alpha}^{\mathrm{s}} \\
u_{\mathrm{r} \beta}^{\mathrm{s}}
\end{array}\right]=\left[\begin{array}{cc}
\hat{\psi}_{\mathrm{r} \alpha} & \hat{\psi}_{\mathrm{r} \beta} \\
\hat{\psi}_{\mathrm{r} \beta} & -\hat{\psi}_{\mathrm{r} \alpha}
\end{array}\right] \cdot\left[\begin{array}{c}
1 / \tau_{\mathrm{r}} \\
\hat{\omega}_{\mathrm{r}}
\end{array}\right]
$$

we have

$$
\hat{\omega}_{\mathrm{r}}=\frac{1}{\left|\hat{\psi}_{\mathrm{r}}\right|}\left(\hat{\psi}_{\mathrm{r} \alpha} u_{\mathrm{r} \beta}^{\mathrm{s}}-\hat{\psi}_{\mathrm{r} \beta} u_{\mathrm{r} \alpha}^{\mathrm{s}}\right)
$$

where $\left|\hat{\psi}_{\mathrm{r}}\right|=\sqrt{\left(\hat{\psi}_{\mathrm{r} \alpha}^{2}+\hat{\psi}_{\mathrm{r} \beta}^{2}\right)}$.

It follows from (13), wheel speed estimation expressions can be calculated by the flux estimator without any integral process. Compared with the traditional observation error of stator current and rotor flux, which directly enters into sliding mode observation with velocity integral part. This paper presented sliding mode observer for wheel speed is more simple, practical and reliable. 


\section{Experimental Results and Analysis}

As can be seen in Figure 1, experimental system architecture is based on sliding mode observer for wheel speed of sensorless electric vehicle. The system's main circuit uses the voltage type SPWM inverter. The speed of in-wheel motor adopt slip frequency control methods, in the process of speed change, the stator current frequency always follows the actual speed of the rotor synchronous lifting or dropping to achieve a smooth speed regulation. Experimental system outer loop is PI speed controller, and the input is deviation between reference speed and estimated speed, the output is current reference value for the synchronous side; for inner loop to complete the current regulatory function, the input is deviation between the current reference value and the measured value, the output is the stator voltage reference value. The in-wheel motor parameters are as following:

$\mathrm{R}_{\mathrm{s}}=0.1 \Omega, \mathrm{R}_{\mathrm{r}}=0.15 \Omega, \mathrm{L}_{\mathrm{s}}=69.9 \mathrm{mH}, \mathrm{L}_{\mathrm{r}}=69.9 \mathrm{mH}, \mathrm{L}_{\mathrm{m}}=68 \mathrm{mH}, \mathrm{J}=0.0586 \mathrm{~kg} \cdot \mathrm{m} 2$.

The reference wheel speed between $0 \sim 0.3$ seconds is ramp-up, between $0.3 \sim$ 0.5 seconds for constant speed 1400rpm, and between $0.5 \sim 0.7$ seconds for even speed reduced to 500rpm, after which the wheel speed maintained constant. The actual wheel speed and the estimated wheel speed are presented in Figure 2 and the estimation error of wheel speed is presented in Figure 3. From Figure 2, it shows the basic agreement by the estimated wheel speed and the actual wheel speed, only in the start time $(<0.08$ seconds) there is a certain chatting and a certain estimation error $(<30 \mathrm{rpm})$ at the wheel speed mutations.

There are still the torque response depicted in Figure 4, the rotor fluxes depicted in Figure 5, and the q-axis current depicted in Figure 6. So the presented sliding mode observer has a good estimate of the wheel speed capability.

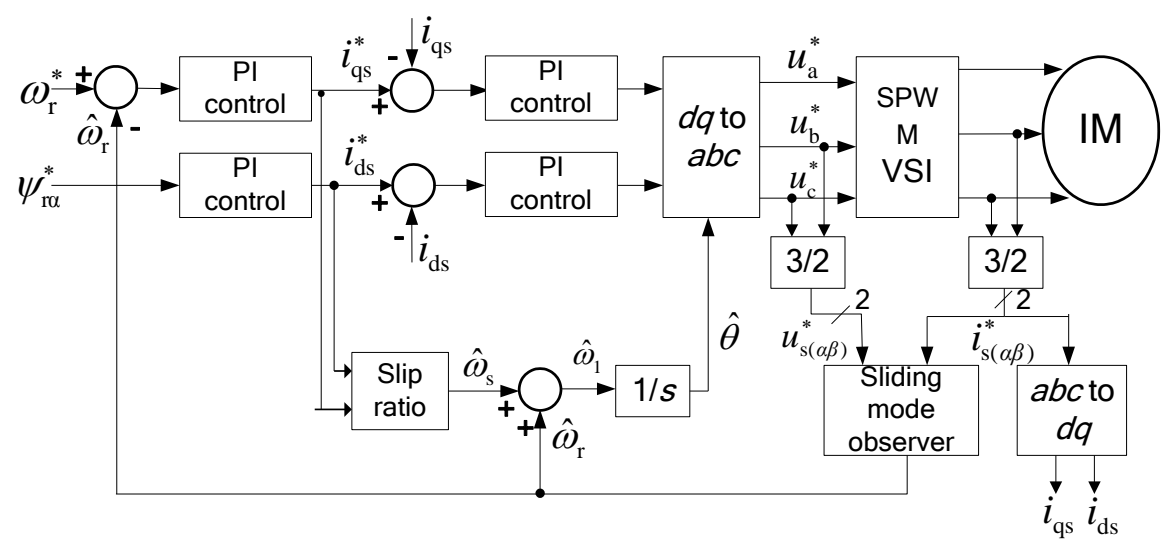

Figure 1. The Sliding-Mode Observer for the Wheel Speed of Sensorless Electric Vehicle 


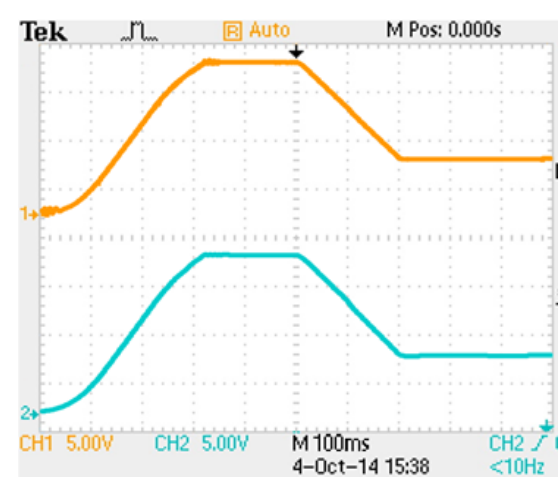

Figure 2. Actual and the Estimated Wheel Speed

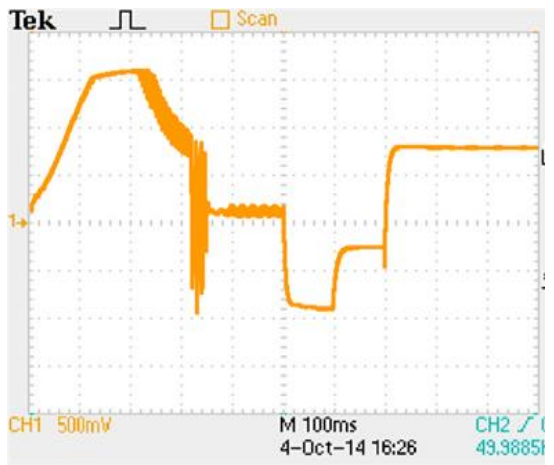

Figure 4. The Torque Response

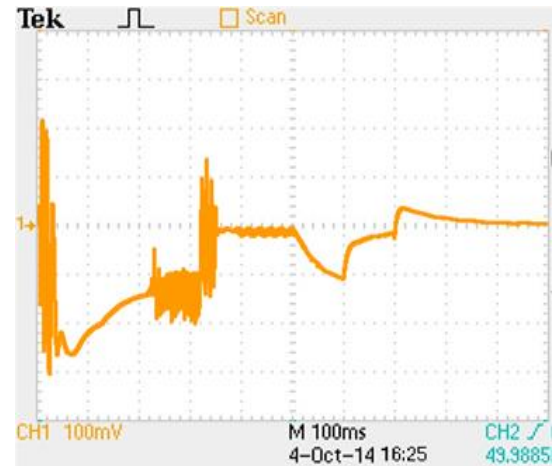

Figure 3. Speed Estimation Errors

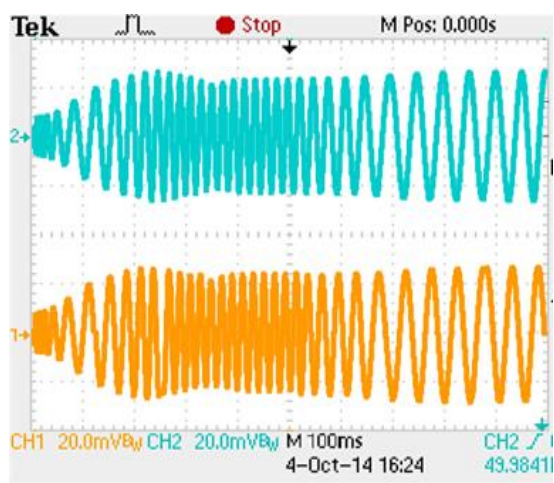

Figure 5. The Rotor Fluxes

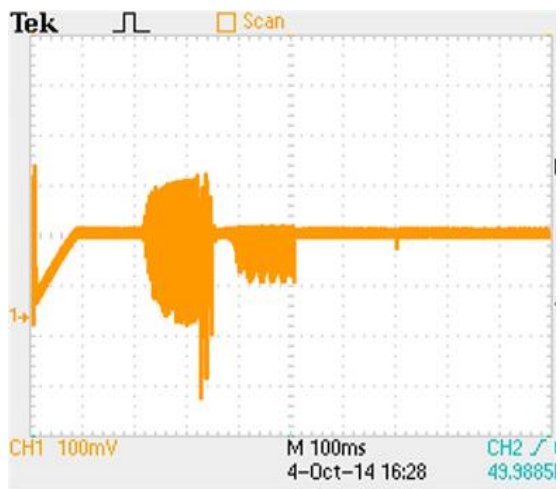

Figure 6. The Q-Axis Current

\section{Conclusion}

This paper presented a novel sliding-mode observer for wheel speed to replace the conventional speed sensors of the distributed-driven electric vehicle with inwheel motors. Compared with conventional SMO, the proposed scheme is more simple, practical and reliable. Observing object is in-wheel motor stator current and rotor flux, through clever design observation equation, at only measure stator currents and voltages can be obtained the wheel speed estimate expressions. Experimental results show that this method can effectively estimate the wheel speed with high accuracy as well as its fast convergence rate onlinely. 


\section{Acknowledgements}

The authors are grateful to the support of the National Natural Science Foundation of China (61203019), the Department of Education (14A032) and Innovative Research Team in Higher Educational Institutions of Hunan Province, and the Natural Science Foundation of Hunan Provincial (no. 13JJ9019).

\section{References}

[1] L. Zhang and G. Ning, "Review of Electrical Vehicles In China", Research on Automotive Industry, vol. 13, no. 12, (2006), pp. 30-33.

[2] Z. Yu, Y. Feng and L. Xiong, "Review of Power Electronics and Science Development Review on Vehicle Dynamics Control of Distributed Drive Electric Vehicle", Journal of Mechanical Engineering, vol. 49, no. 8, (2013), pp. 105-114.

[3] L. Xiong, Z. Yu and W. Jiang, "Research of vehicle stability control of 4WD electric vehicle based on longitudinal force control allocation", Journal of Tongji University, vol. 38, no. 3, (2010), pp. 417$421+426$.

[4] A. Haddoun, M.E.H. Benbouzid, D. Diallo and R. Abdessemed, "Modeling, Analysis, and Neural Network Control of an EV Electrical Differential", IEEE Transactions on Industrial Electronics, vol. 55, no. 6, (2008), pp. 2286-2294.

[5] K Yi. T. Chung and J. Kim, "An investigation into differential braking strategies for vehicle stability control", Proceedings of the Institution of Mechanical Engineers, Part D- Journal of Automobile Engineering, vol. 217, no. 12, (2003), pp. 1081-1093.

[6] N. Mutoh, Y. Hayano and H. Yahagi, "Electric braking control methods for electric vehicles with independently driven front and rear wheels", IEEE Transactions on Industrial Electronics, vol. 54, no. 2, (2007), pp. 1168-1176.

[7] J. M. Wang and R. G. Longoria, "Coordinated and Reconfigurable Vehicle Dynamics Control", IEEE Transactions on Control Systems Technology, vol. 17, no.3, (2009), pp. 723-732.

[8] D. Amir, B. H. Alireza and Z. Cao, "Intelligent Sensorless ABS for In-Wheel Electric Vehicles", IEEE Transactions on Industrial Electronics, vol. 61, no. 4, (2014), pp. 1957-1969.

[9] Y. Shen and X. Wu, "Multiplex Neural Network Control of Four-wheel Drived Electric Vehicle", Automotive Engineering, vol. 26, no. 4, (2004), pp. 458-460.

[10] J. Yang, F. Li and H. Ding, "Vehicle Yaw Stability Control Based on Generalized Predictive Control", Transaction of the Chinese Society for Agricultural Machinery, vol. 43, no. 1, (2012), pp. 1-5+36.

[11] Y. Zhao and J. Zhang, "Stability Control for a Four-Motor-Wheel Drive Electric Vehicle Based on Sliding Mode Control", Journal of Shanghai Jiaotong University, vol. 43, no. 10, (2009), pp. 1526-1530.

[12] D. Amir, Z. Cao and A. Kapoor,"Intelligent sensorless ABS for regenerative brakes", Proceedings of the International Electric Vehicle Conference (IEVC), Greenville, SC, (1995) March 4-8.

[13] M. Kazemi and K.H. Shirazi, "Handling enhancement of a sliding-mode control assisted four-wheel steer vehicle", Proceedings of the Institution of Mechanical Engineers, Part D: Journal of Automobile Engineering, vol. 226, no. 2, (2012), pp. 234-24.

\section{Author}

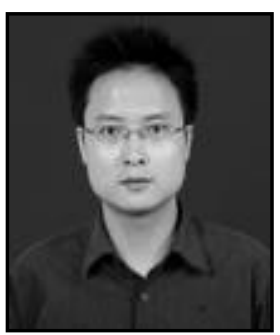

Xizheng Zhang, he received the B.S. degree in electrical engineering, the M.S. degree in circuits and systems and control engineering in 2000, 2003 and 2010, respectively, from Hunan University, China, He is also with the School of Computer and Communication, Hunan Institute of Engineering, China. His research interests include control theory and its application. 
International Journal of Control and Automation

Vol. 10, No. 2 (2017) 